\title{
Batch Mode Job Scheduling Scheme (BJSS) for Clusters in Underwater Wireless Sensor Network (UWSN)
}

\author{
Pranay Mondal \\ School of Electronics, \\ KIIT University \\ Bhubaneswar, India
}

\author{
Nirmal Kumar Rout \\ School of Electronics, \\ KIIT University \\ Bhubaneswar, India
}

\begin{abstract}
Cluster selection of Underwater Wireless Sensor Network (UWSN) is an important factor for achieving lowest energy consumption and thus makes it more energy efficient. This energy efficiency also enhances the lifespan of the network. In this paper, we propose a Batch mode Job Scheduling Scheme (BJSS) technique for selection of the cluster. The BJSS is a standout among the most mainstream individuals for cluster selection. The selection of these clusters are based upon criteria such as number of jobs allocated to the cluster head $(\mathrm{CH})$, numbers of clusters available for doing that job, time consumption for doing the allocated job and energy of that cluster. For assessment of cluster selection, several threshold values, to represent practically possible energy consumptions are introduced. This proposed technique is verified by the simulation results. By using this technique UWSN is more energy-efficient and long lifespan of the battery or power source.
\end{abstract}

\section{Keywords}

Multi-criteria decision making, MCDM, PROMETHEE II, Wireless Sensor Network, WSN.

\section{INTRODUCTION}

Underwater Wireless Sensor Network (UWSN) is a gathering of very small-scale smart sensing devices. These very smallscale sensing devices are positioned arbitrarily in different observing regions. UWSN, one can explore the underwater applications. Such as Data collection of oceanographic, Natural disasters (e.g. Tsunami and seismic monitoring), Ecological balance application (e.g. pollution and biological and water quality monitoring), underwater military surveillance in a large ocean, Navigation assistance, etc. These sensing devices collect information from observing regions and transfers it towards the cluster head with the support of multi and single hop communication [1], [2].

UWSN normally contains low-priced devices that can communicate with one another by utilizing multi-hop communication. Every node of UWSNs is called a sensor cluster, which comprises of limited memory, implanted processors and is regularly worked with battery.

However, because of random allocation of sensing tasks, few clusters unlooses massive energy. The misbalanced energy circumstance generates an energy hole in this UWSN network (Fig. - 1). In UWSN, the energy hole reflects that in that region all the cluster died due to power failure.

So, to reduce the energy hole problem, we have to schedule job allocation in the network more efficiently. Most significantly, clusters are commonly installed in a large area.
These clusters are normally active and deposal when battery is ending.

Cluster selection talks about the optimization for selecting proper sets of clusters for working on the network with minimum energy consumption and most cost effective. Here we have taken consideration of energy consumption, threshold value and coverage of each cluster.

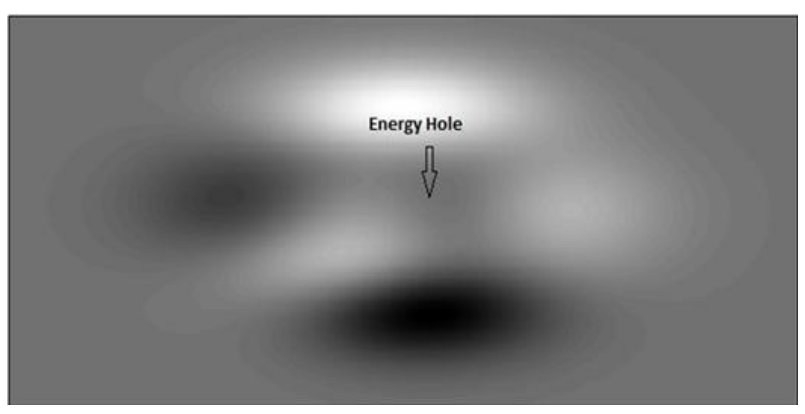

Fig. - 1. Energy hole in the UWSN network

Among the numerous node selection technique and cluster head $(\mathrm{CH})$ node selection technique, Nguyen [3] done this $\mathrm{CH}$ node selection based only on the distance. From the LEACH protocol [4], he proposed DB-LEACH and then next to DBEA-LEACH. This DBEA-LEACH establishes new threshold value for sensor node based on the distance and position of the sensor node from the base station and each other. It also introduced about the initial and current energy to node determination probability.

It ought to be noticed that the DBEA-LEACH protocol is considered only about the distance and position of the sensor node. However, it does not talk about the energy consumption and cost perspective.

In this paper, we proposed a new technique for cluster selection on the network. For doing these here few aspects like energy consumption, threshold value and last but not the coverage factors are also considered. By the use of BJS algorithm, all the considerations are taken care of equally.

The formations of this paper are as per the following. Section II tells about mathematical equations used for BJSS. In section III introduces proposed BJSS technique. Section IV discusses about job allocation and algorithm of BJSS. Followed by performance evaluations in section $\mathrm{V}$ and finally section VI presents conclusions and future work. 


\section{MATHEMATICAL EQUATIONS FOR BJSS}

This section briefly describes about energy loss of the sensor nodes for data transmitting and data receiving process in the cluster. Next portion describes effective cluster size calculation technique. According to effective cluster size, the whole network is divided into effective clusters.

\subsection{Data Communication Model}

The energy consumption by each cluster for single message transmission is represented by the linear equation [1]

$$
E\left(d_{i}\right)=\left(\alpha_{t+} \alpha_{d} * \gamma^{n}\right) L_{i}
$$

Where $\alpha_{t}$ is the energy loss per bit by the transmitter electronics circuit, and $\alpha_{d}$ is the dissipated energy in the transmitter op-amp. Transmission range is $\gamma$. The parameter ' $n$ ' is power index for the channel path loss of the antenna. $\mathrm{Li}$ is the message size which is transmitted by each sensor cluster. Receiving energy loss of each cluster is represented by the following formula.

$$
E\left(d_{r}\right)=\left(\alpha_{r} L_{i}\right)
$$

Where, $\alpha_{r}$ is energy per bit, which is consumed by the receiver's electronics circuit used by the cluster. $L_{i}$ message size, which is received by each sensor cluster.

So the energy gain by the cluster is -

$$
E=E\left(d_{i}\right)-E\left(d_{r}\right)
$$

\subsection{Optimal Cluster Size calculation}

The cluster head life time depends both on inter-cluster communication cost and intra-cluster communication cost. Inter cluster communication cost is the total energy loss for data transmission from cluster head to base station. On the other hand, intra-cluster communication cost depends on the energy loss for data transmission from cluster member nodes to cluster head. If cluster size is increased then the inter cluster communication cost is decreased, but intra-cluster communication cost is increased. On the other hand, if cluster size is very small then intra-cluster communication cost is decreased, but inter-cluster communication cost is increased. If a cluster contains $\mathrm{N}$ numbers of nodes, then inter-cluster communication cost is given by [2]

$$
I_{\text {intar }}=\sum_{1}^{N}\left(E\left(d_{i}\right)+E\left(d_{r}\right)\right)
$$

If a cluster head is located $\emptyset$ distance from the base station and after data gathering, gathered data packet size of cluster head is $L_{\text {age }}$ then inter cluster communication cost for single hop communication.

$$
I_{\text {inter }}=\left(\alpha_{t}+\alpha_{d} \emptyset^{N}\right) L_{a g e}
$$

The total inter and intra cluster communication cost is

$$
I_{\text {total cost }}=I_{\text {intra }}+I_{\text {inter }}
$$

After deployment of the sensor nodes in the monitoring environment they will determine their position via any existing self-localization technique [5-9]. After collection of information from the nodes of the clusters, the base station chooses the clusters such that the $I_{\text {total cost }}$ is minimum in
UWSN. Therefore, in batch mode job scheduling scheme, clusters are shaped such that there are optimal communication energy losses.

\section{PROPOSED BATCH MODE JOB SEHEDULEING SCHEME}

In the proposed batch mode job scheduling (BJSS) the clusters are generated in the initial steps. Then the job scheduling among the member node is carried out by each cluster head. In BJSS I have defined the few parameters for my implementation are as follows:

Definition 1: Supercede number of a job are defined as the number of jobs get allocated before the particular job get allocated.

Definition 2: An inert cluster is a cluster that is made inactive by the allocation of a particular cluster for a job.

Definition 3: A candidate cluster $m^{\#}$ is a cluster $m^{\#} \in M$ which is available for allocation for a job $\left(M-I=m^{\#}\left[m^{\#} \in\right.\right.$ $\left.M, m^{\#} \in I\right)$ where $\mathrm{I}$ is non-candidate cluster.

Definition 4: A non-candidate (I) cluster is a cluster, which is either already allocated, or inert, or dead and is not available in the allocation of a job.

For a task requiring $\mathrm{n}$ number of clusters to be allocated, clusters are allocated one at a time. Before allocation starts the strategy checks the mesh for the adequate availability of active nodes required for the job under any cluster. If the availability satisfies the requirement, allocation process for the job starts. Each cluster of a task is allocated on the basis of the highest energy clusters available in a mesh. The first cluster for a task is allocated on the basis of the highest energy cluster available in the entire mesh. For the other cluster of the tasks, the clusters are allocated from a certain region of the mesh. This region of the mesh from which a cluster is to be considered for allocation is defined by the previously allocated cluster of the same task. The highest energy cluster from the pre-defined region of the mesh is allocated next to the task. In the given algorithm a cluster already allocated does not allow a fixed number of clusters around it to be allocated for the clusters of the same job to provide better coverage. When the mesh gets exhausted, i.e. all the clusters are non-candidate clusters, then all the inert clusters, if available, are turned active and allocation process continues. This process continues until all the clusters are required for the job gets allocated. For this job allocation cluster is also chooses accordingly with the no of nodes available on that cluster.

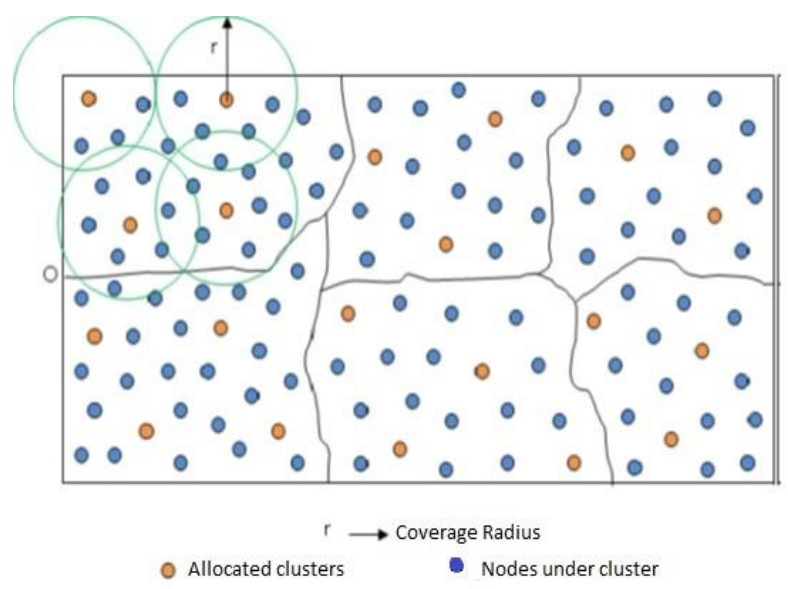

Fig. - 2. Job allocation In UWSN 
The regions of the cluster from which clusters are to be considered for allocation for a job, discussed earlier, depends on two factors:

1. On how many nodes are present in that cluster for completing the schedule job.

2. On coverage parameter.

When a cluster ' $\mathrm{P}$ ' for a job gets allocated coverage numbers of clusters around the cluster P are made inert. It is done so that the next cluster for the job gets allocated at a considerable distance from the present node P. This is done to keep the clusters for a job allocated evenly throughout the mesh. This helps in the uniform assimilation of information from the entire range of the information (Fig. 4).

Each task has a task-head, which points to the first cluster allocated for the task. This cluster subsequently points to the next cluster allocated for the task and so on, giving it a structure of a linked list.

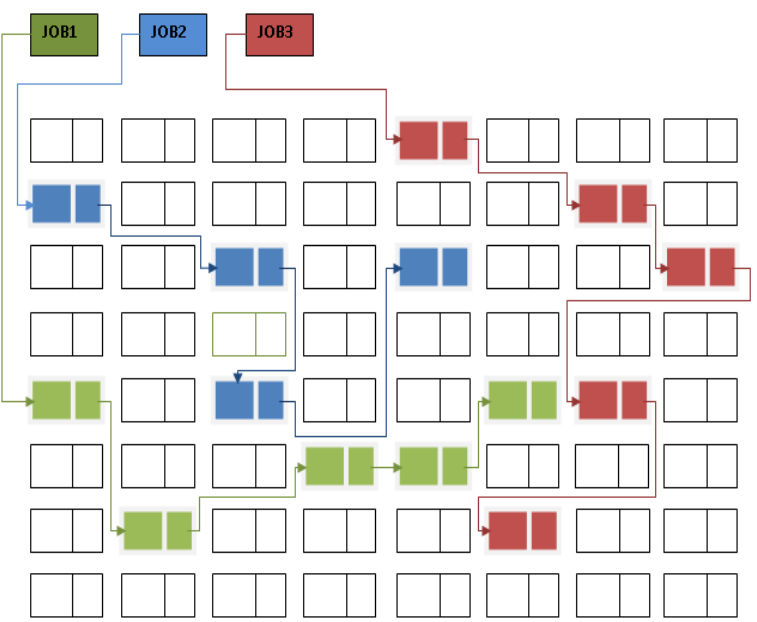

Fig. - 3.Task allocation in a mesh by linked technique

\section{ALGORITHM AND JOB ALLOCATION PROCESS}

\subsection{Job allocation}

If the availability of the number of clusters in the mesh is lesser than the number of clusters required for a job, the job gets appended to the waiting queue in a cluster head. The jobs in the waiting queue do not stop the allocation of the incoming jobs until the input parameter MAX_SUPERCEDE number of jobs gets allocated before the job(s) waiting at the head of the waiting queue. Each job in the waiting queue has a parameter to record the number of jobs arriving after it that gets allocated (supercede). The moment the parameter supercede for the job(s) at the head of the queue becomes equal to or greater than the parameter MAX_SUPERCEDE, further allocation of the incoming jobs is suspended. The jobs with parameter supercede greater than or equal to the parameter MAX_SUPERCEDE are given higher priority than the other jobs in the waiting queue. If there are more than one number of job in the class of these higher priority jobs, the task with the smallest job in the queue is allocated first, and gradually all the other higher priority jobs are allocated.

As a job is deallocated from the mesh in cluster head, the possible jobs are searched in the waiting queue that could be allocated. The random allocation process of the jobs in the waiting queue is suspended if any of the job(s) at the head of the waiting queue has the parameter supercede greater than or equal to MAX_SUPERCEDE. The suspension prevails until the higher priority job(s) of the waiting queue get allocated.

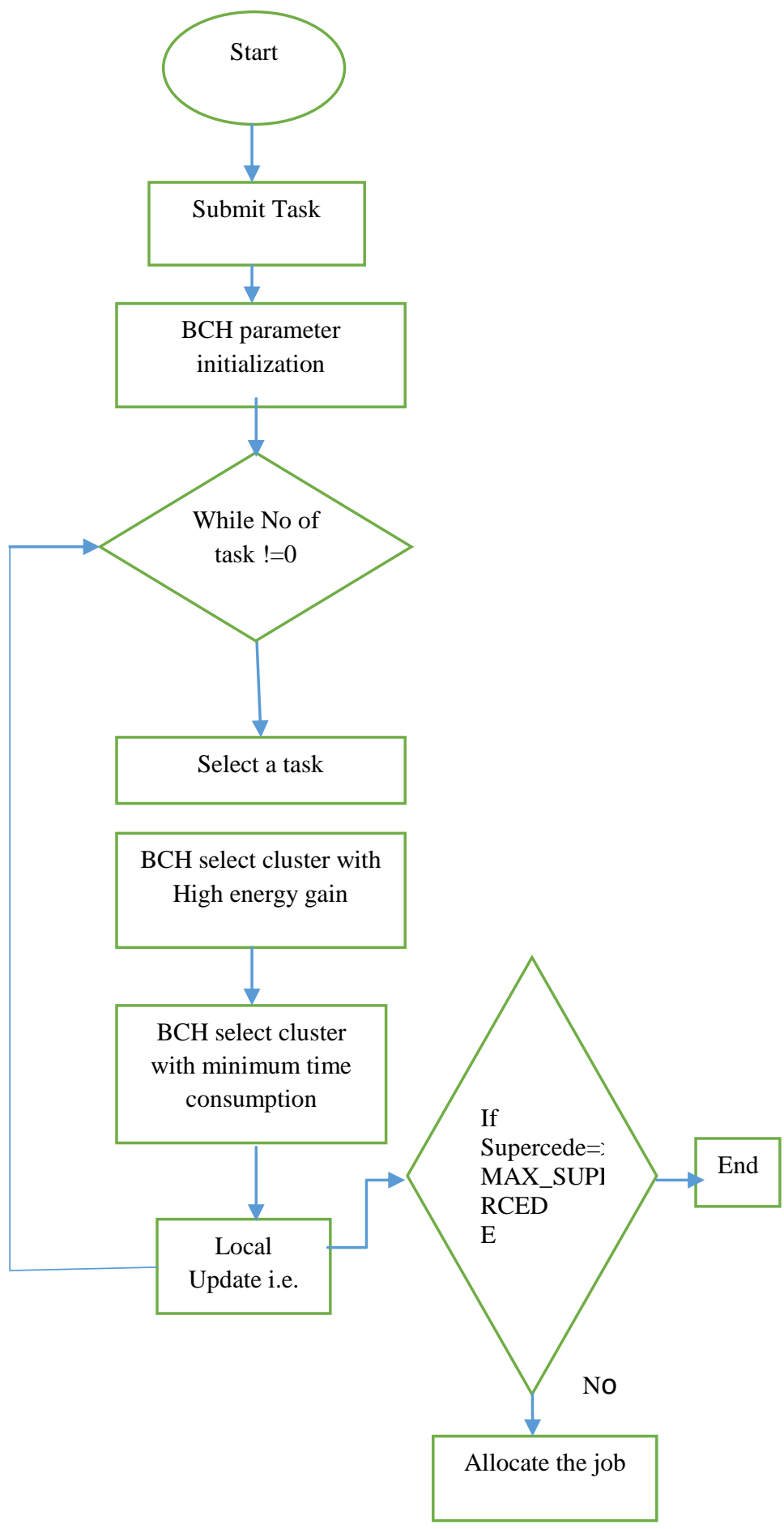

4.1.1 Algorithm for BJSS

Another parameter, MAX_SUPERCEDE value takes care that none of the jobs get starved. When MAX_SUPERCEDE numbers of jobs get allocated before a job or group of jobs waiting in the queue, further allocation stops until the job(s) with higher priority in the waiting queue gets allocated. Jobs from this high priority queue get allocated with respect to their nodes under that cluster and distance of the cluster from the base station. Thus the jobs that have been waiting for a long time for their allocation, gets allocated. Smaller jobs would require lesser number of clusters for allocation and are 
allocated sooner than the jobs requiring more number of clusters as the number of clusters required for the job is available by the deallocation of the already allocated jobs. This decreases the overall average waiting time of the jobs (jobs in the high priority queue and the jobs in the normal queue) increasing the efficiency of the network. For the scheduling strategy, once the supercede number of the job at the top of the waiting queue reaches MAX_SUPERCEDE no other job behind it even with its supercede number equal to MAX_SUPERCEDE are allowed to be allocated thus increasing the average waiting time of the system. In the proposed strategy due to no of nodes and distance between the cluster head and the base station, the smaller jobs behind the biggest job with the same supercede number gets allocated first, thus reducing the average waiting time. The proposed strategy guarantees the network to be starvation free. This is due to the fact that a job which is in the waiting queue will eventually follow the deallocation of previously allocated jobs in the cluster if node is available under the cluster to do the work. This avoids starvation.

\section{PERFROMANCE EVALUATION}

A series of simulation work is done to select the optimum values for my proposed work. The mesh network is first divided into 3 clusters. So by this clusters my proposed algorithm will choose the right cluster for the work according to the conditions that have been discussed.

Here we have taken the following values-

No of task is taken 3. So, accordingly supercede and MAX_SUPERCEDE values are 0 and 3 .

Here the energy loss per bit by the transmitter electronic circuit i.e. $\alpha_{t}$ is $10 \mathrm{~nJ}$ and dissipated energy in the transmitter op-amp i.e. $\alpha_{d}$ is $5 \mathrm{pJ}$. The value of transmission range i.e. $\mathrm{Y}=1$. Here antenna power index for the channel path loss which is denoted as $\mathrm{n}$ is 2 . Energy loss per bit i.e. $\alpha_{r}$ will be $3 \mathrm{pJ}$. Message length $\left(L_{i}\right)$ is taken as 3 .

For the calculation of minimum time consumption, we assume that on the 3 clusters there are 2,3,5 nodes $(\mathrm{N})$ and their distance $(\varnothing)$ are 3,4,6 unit respectively.

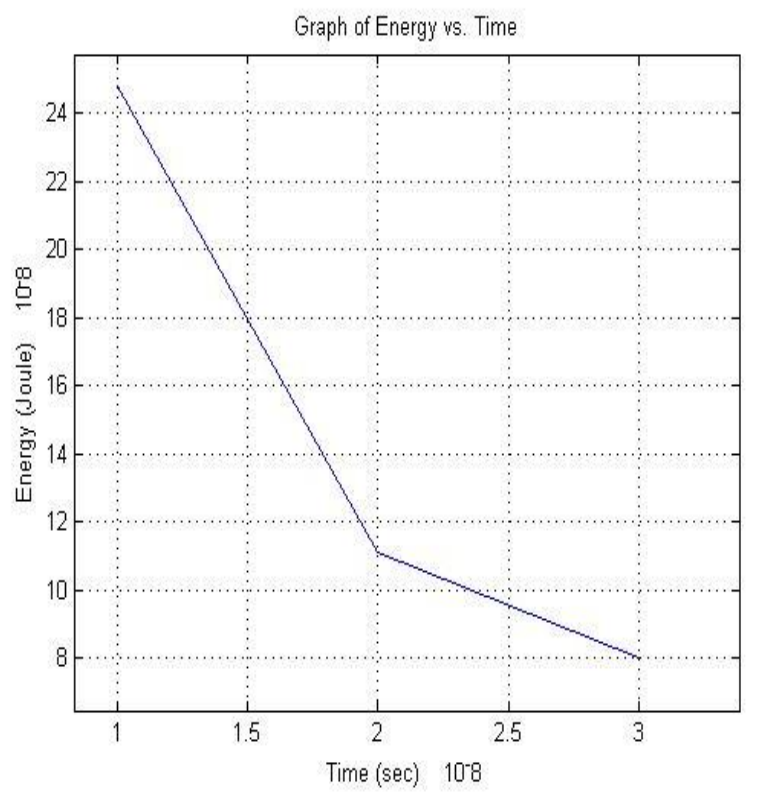

Fig. 4 - Energy utilization of UWSN clusters with minimum time consumption

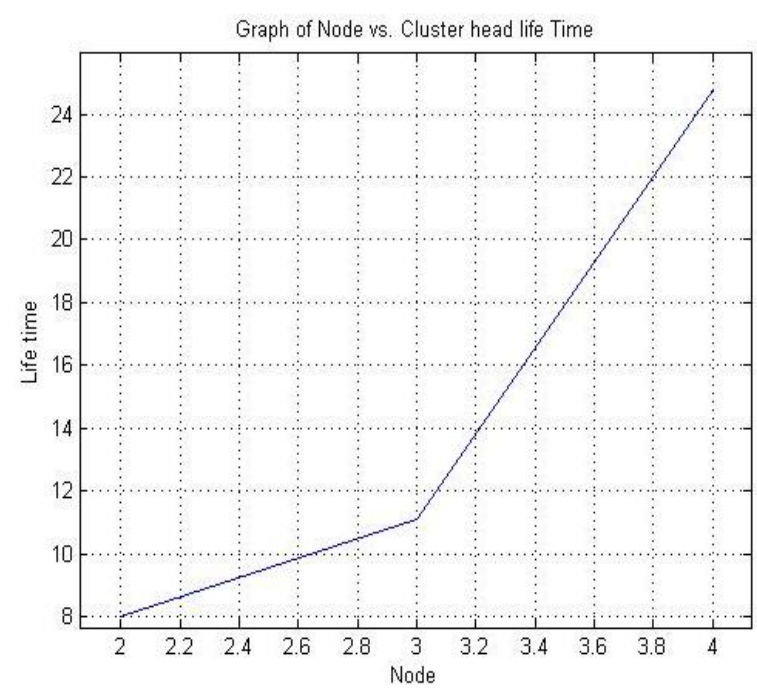

Fig. 5 - Network life time with no of nodes present in that cluster

\section{CONCLUSION AND FUTURE WORK}

In this paper, we proposed a technique for selecting the best suitable cluster for a sensor network. For this, we have used BJSS. By doing this technique, we can select a better cluster, which has less consumption of energy, more cost-effective and prolonging the network lifetime.

For the evaluation, MATLAB Simulation tool is used to visualize the results of this technique. From the simulation results show it outperforming other cluster selection techniques in respect of consumption of energy, long lifetime and cost-effectiveness.

Even though our proposed technique can accomplish performance enhancements, stills many aspects of this technique need to be scrutinized by using heterogeneous scenarios and restraints. Furthermore, there are many other factors, which affect consumption of energy, a threshold value of the network could be investigated more.

\section{REFERENCES}

[1] Indrajit Banerjee, Prasenjit Chanak, Biplab k. sikdar, Hafijur Rahaman, "EERIH: Energy Efficient Routing via Information Highway in Sensor Network," IEEE International conference on emerging trends in Electrical and Computer technology, March 23rd and 24th 2011, kanyakumari, India.

[2] Indrajit Banerjee, Prasenjit Chanak, Biplab k. sikdar, Hafijur Rahaman, "EER: Energy Efficient Routing in Wireless Sensor Networks," IEEE International Technology Symposium 2011, 14 - 16 January, IIT kharagpur, India.

[3] Tri Gia Nguyen, Chakchai So-In, Nhu Gia Nguyen, "Two Energgy-Efficient Cluster Head Selection Techniques Based on Distance for Wireless Sensor Networks," International Computer Science and Engineering Conference (ICSEC) 2014.

[4] W. B. Heinzelman, A.P. Chandrakasan, and H Balakrishnan, "An Application-Specific Protocol Architecture for wireless Microsensor Networks," IEEE Trans. On Wireless commun., pp. 660-670, 2002. 
[5] Caruso, S. Chessa, S. De, and A. Urpi, "Gps-free coordinate assignment and routing in wireless sensor networks," in Proc. IEEE INFOCOM, Miami, USA, 2005, pp. 150-160.

[6] Mirza and C. Schurgers, "Motion-aware self-localization for underwater networks," in Proc. of third ACM international workshop on Underwater Networks, San Francisco, California, USA, 2008, pp. 51-58.

[7] D. Mirza and C. Schurgers, "Energy-efficient ranging for post-facto self-localization in mobile underwater networks," IEEE J. Sel. Areas Commun., vol. 26, no. 9, pp. 140-152, Dec 2008.

[8] Tan and J. Li, "Cooperative positioning in underwater sensor networks," IEEE Transactions on Signal Processing, vol.58, no.11, pp.5860-5871, Nov. 2010.

[9] T. Bian, R. Venkatesan, and C. Li, "An improved localization method using error probability distribution for underwater sensor networks," in Proc IEEE International Conference on Communications (ICC), Cape Town, South Africa, May 2010, pp. 1-5. 